\title{
BMJ Open Neighbourhood socioeconomic position and risks of major chronic diseases and all-cause mortality: a quasi- experimental study
}

\author{
Daniel Kim, ${ }^{1,2}$ Richard H Glazier, ${ }^{3,4,5}$ Brandon Zagorski, ${ }^{6,7}$ Ichiro Kawachi, ${ }^{8}$ \\ Philip Oreopoulos ${ }^{9}$
}

To cite: Kim D, Glazier RH, Zagorski B, et al. Neighbourhood socioeconomic position and risks of major chronic diseases and allcause mortality: a quasiexperimental study. BMJ Open 2018;8:e018793. doi:10.1136/ bmjopen-2017-018793

- Prepublication history for this paper is available online. To view these files, please visit the journal online (http://dx.doi org/10.1136/bmjopen-2017018793).

Received 24 July 2017 Revised 22 December 2017 Accepted 3 January 2018
Check for updates

For numbered affiliations see end of article.

Correspondence to

Professor Daniel Kim; d.kim@northeastern.edu

\section{ABSTRACT}

Objectives This study estimated the health impacts of neighbourhood socioeconomic position (SEP) among public housing residents. Because applicants to public housing were assigned to housing projects primarily based on factors other than personal choice, we capitalised on a quasirandom source of variation in neighbourhood of residence to obtain more valid estimates of the health impacts of neighbourhood SEP.

Design Quasiexperimental study.

Setting Greater Metropolitan Toronto area, Canada. Participants Residents (24019-28858 adults age $\geq 30$ years in 1994 for all outcomes except for asthma, for which the sample was expanded to 66627 individuals age $\geq 4$ years) of public housing on 1 January 1994 .

Outcome measures Incident hypertension, diabetes, asthma, and acute myocardial infarction (MI) and all-cause mortality between 1 January 1994 and 31 December 2006. We used multivariate Cox proportional hazards models to estimate hazard ratios (HRs) for the associations between the quartile of census tract-level SEP and the risk of diagnosis of each health outcome as well as death from any cause.

Results Living in a public housing project in the second highest neighbourhood SEP quartile (Q3) was associated with lower hazards of acute $\mathrm{Ml}(\mathrm{HR}=0.76,95 \% \mathrm{Cl} 0.54$ to 1.07; $\mathrm{P}=0.11$ ), incident asthma ( $\mathrm{HR}=0.80,95 \% \mathrm{Cl} 0.67$ to $0.96 ; \mathrm{P}=0.02)$ and all-cause mortality $(\mathrm{HR}=0.86,95 \% \mathrm{Cl}$ 0.73 to $1.01 ; P=0.06$ ) compared to living in the lowest neighbourhood SEP quartile (Q1), although only the trend for incident asthma reached statistical significance ( $P$ for trend $=0.04$ ). By contrast, the associations corresponding to living in the highest versus lowest quartile of median household income (Q4 vs Q1) were neither consistent in direction nor significant. The inconsistent associations may partly be attributed to selection and status incongruity. Conclusion This study provides new evidence compatible with protective influences of higher neighbourhood SEP on health outcomes, particularly asthma.

\section{INTRODUCTION}

Varying levels of neighbourhood socioeconomic position (SEP) may help to account for observed variations in population health, over
Strengths and limitations of this study

- Strengths of our study include its reliance on comprehensive administrative data including health outcomes based on provincial health and vital statistics databases to enhance validity and enable inclusion of all eligible adult residents of public housing in a large metropolitan area.

- We further capitalise on a novel quasiexperimental design to help minimise bias due to confounding.

- This study encompasses a wider set of major chronic disease outcomes and mortality than previously investigated in a single study of neighbourhood effects.

- Moves into or out of the census tracts as well as differential attrition due to deaths prior to baseline could contribute to selection bias.

- Our study findings may not necessarily generalise beyond those living in public housing in urban areas, particularly across much wider ranges of neighbourhood and individual socioeconomic position.

and above personal factors such as individual income and education. ${ }^{1}$ Neighbourhood SEP is plausibly a determinant of amenities and resources including the local food environment (eg, density of supermarkets), built environment (eg, street connectivity), presence of tobacco and alcohol vendors (ie, tobacco outlets) and air quality. ${ }^{2}$ In turn, such neighbourhood attributes may mediate the health impacts of neighbourhood SEP by influencing individual health behaviours and physical health and ultimately shaping the risks of chronic health conditions. ${ }^{2-4}$

Growing empirical evidence from epidemiological studies supports associations between neighbourhood socioeconomic environments and the incidence of chronic diseases/conditions and mortality. Studies have demonstrated associations between neighbourhood SEP and all-cause mortality, with relative risks between 1.1 and 1.8 for 
higher versus lower levels of area-based socioeconomic deprivation, after taking into account individual SEP. ${ }^{5-7}$ Furthermore, studies have linked neighbourhood SEP to major chronic conditions including depression, diabetes, and hypertension. ${ }^{4-10}$

Nonetheless, many of these reported findings to date have been plagued by major threats to internal validity including residual confounding and endogeneity bias. These threats have prompted the need for more rigorous methodological approaches (eg, instrumental variable and propensity score methods) and study designs (eg, natural experiments/quasiexperimental designs) to attenuate such sources of bias. ${ }^{11-13}$ Moreover, a large proportion of these studies has been cross-sectional in design, making causal inferences on the basis of temporality more tenuous.

To reduce endogeneity bias due to confounding, several studies investigating the impacts of neighbourhood factors on non-health outcomes have exploited the quasirandom variation in neighbourhood of residence (eg, such as generated by waiting list lotteries for public housing residents) and have implemented experimental designs. For example, Rosenbaum et $a l^{14}$ and Popkin et $a l^{15}$ used families from public housing waiting lists in Chicago to investigate outcomes of parents and children for those who moved to less-segregated suburbs. In the Moving to Opportunity experiment, families living in public and assisted housing in high-poverty inner city neighbourhoods were randomly selected to receive housing vouchers to move to lower-poverty areas. ${ }^{1617}$

In Toronto, Canada, until the mid-1990s, applicants on the Metropolitan Toronto Housing Corporation (MTHC) waiting list were assigned to public housing projects according to factors other than personal choice, primarily based on financial need. ${ }^{18}$ Families who reached the top of the waiting list were offered accommodation according to the first available unit with the requested number of bedrooms. Families in subsidised housing could request a transfer based on a change in employment location or family size, but moving between projects on the basis of poor neighbourhood environments was not permitted. In a previous study, Oreopoulos ${ }^{18}$ exploited this quasirandom source of variation in neighbourhood of residence to obtain more valid estimates of the impacts of neighbourhoods on labour market outcomes.

In the present study, we capitalised on the presence of quasiexperimental variation by drawing on census and provincial health administrative data for public housing residents in the metropolitan Toronto area to estimate the impacts of neighbourhood SEP on the risks of several major chronic diseases and conditions (heart attack, diabetes, hypertension and asthma) as well as the risk of all-cause mortality. Based on previous literature, we hypothesised that higher neighbourhood SEP would have protective influences on the risks of developing these diseases/conditions and all-cause mortality.
MATERIALS AND METHODS

\section{Study population used}

The study population consisted of residents (adults aged $\geq 30$ years in 1994 for all outcomes except for asthma, for which the sample was expanded to include individuals aged $\geq 4$ years in 1994) of MTHC public housing projects on 1 January 1994. There are 106 uniquely identified MTHC family housing projects (figure 1). Each project is associated with a six-digit postal code (with approximately three-quarters of public housing residents being located at addresses with unique postal codes, often single buildings) and census enumeration area (EA; each containing approximately 400-600 individuals). The projects vary considerably in size and are located in census tracts (CT; each containing from 2500-8000 individuals, and an average of 4000 individuals) that encompass a wide range of percentages below the low-income cut-off (LICO), representing low-income to middle-income neighbourhoods (figure 1).

Using postal codes that match uniquely to public housing projects, we identified residents of housing projects through the provincial Registered Persons Database (RPDB). The RPDB for the province of Ontario contains the historical residential addresses, unique provincial health insurance number, date of birth, and time of address changes for each individual in Ontario. The Postal Code Conversion File Plus from Statistics Canada was also employed to identify the census tract in which the resident lived based on the postal code.

\section{Main predictor variables}

The primary exposure was neighbourhood SEP as measured by median household income for the census tract in which the resident lived on 1 January 1994. Each of these socioeconomic measures was derived by taking the value from the Canada 1996 Census and then analysed in separate models as quartile categorical variables.

In addition, a combined SEP measure was constructed based on six income/wealth, education and occupational indicators at the 1996 census tract level (log of median household income, percentage below LICO, log of median value of housing units, percentage with less than high school education, percentage working class, and percentage not in the labour force) ${ }^{19}$ This measure was derived by standardising the values corresponding to the constituent indicators and then by taking their mean.

In sensitivity analyses, we further examined the robustness of the findings when the primary exposure was neighbourhood SEP as measured by the percentage below the LICO for the census tract in which the resident lived.

\section{Health outcomes analysed}

Public housing residents were linked by their unique health insurance numbers within the RPDB to the Ontario Health Insurance Plan (OHIP) database and the Discharge Abstract Database (DAD). The OHIP database includes International Classification of Disease (ICD) diagnostic codes for the condition/disease the 


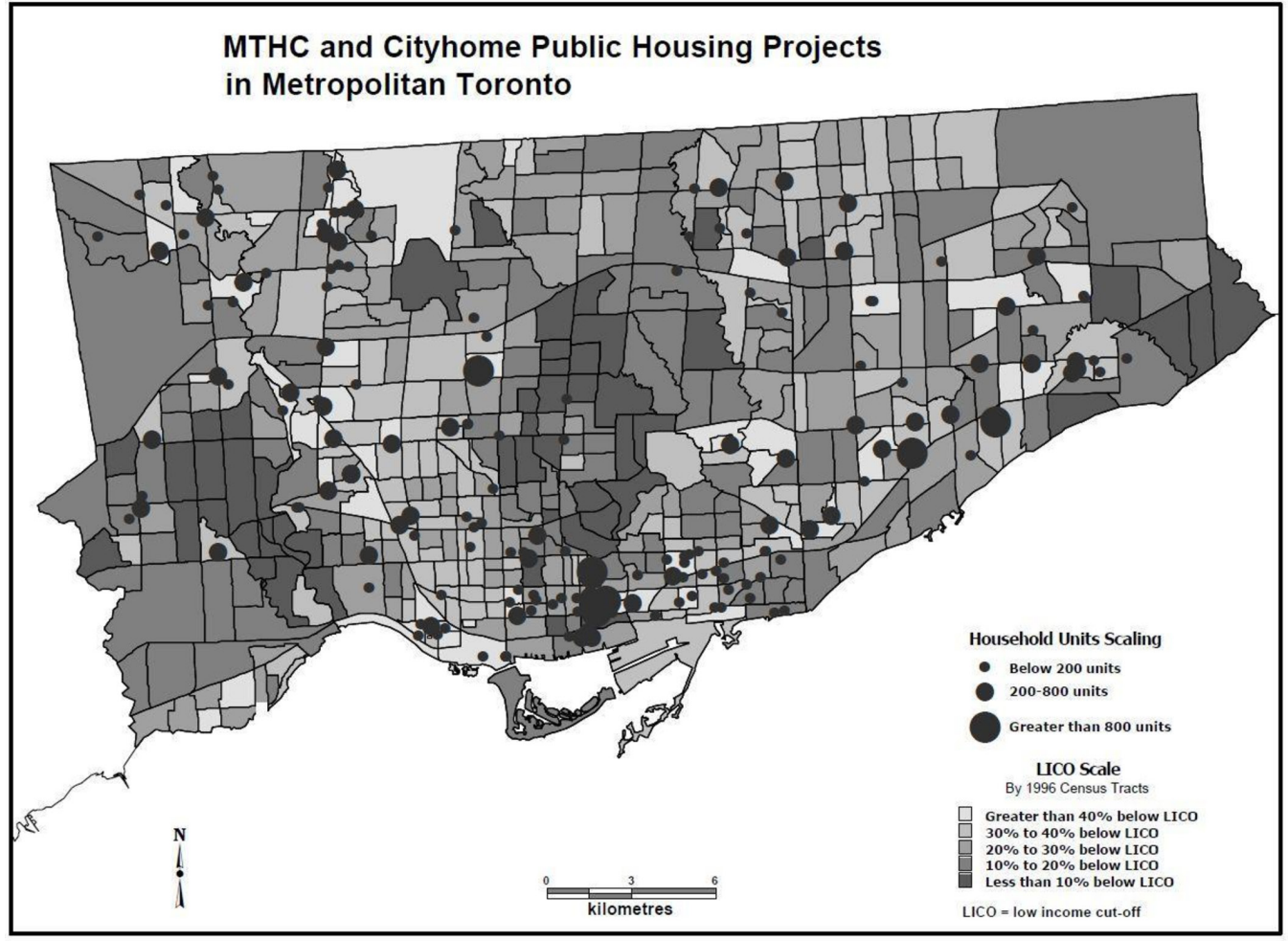

Figure 1 Map of public housing projects in Metropolitan Toronto with Low-Income Cut-Off (LICO) scale. MTHC, Metropolitan Toronto Housing Corporation.

patient was seen for by a physician. The DAD from the Canadian Institute for Health Information contains up to 16 major diagnoses for acute care hospitalisations. Only the most responsible diagnosis field was used to identify diagnoses from the DAD. Incident primary diagnostic health outcomes identified through $\mathrm{OHIP} /$ the DAD consisted of hypertension (ICD-9 codes 401-405, or after 2002, ICD-10 codes I10-I13 or I15), diabetes (ICD-9 code 250, or after 2002, ICD-10 codes E10, E11, E13 or E14), asthma (ICD-9 code 493, or after 2002, ICD-10 codes J45 or J46) and coronary heart disease (acute myocardial infarction (MI) ICD-9 codes 410, 411, 413 or 428, or after 2002, ICD-10 codes I20, I21 or I50), diagnosed during the period between 1 January 1994 and 31 December 2006. For enhanced accuracy for OHIP diagnoses, two claims with a diagnosis were required, ${ }^{20}$ while only one admission with that diagnosis was required for DAD data. All-cause mortality occurring during the same time period was further analysed as an independent health outcome.

\section{Factors controlled for in statistical models}

Area-level model covariates consisted of the mean household income of the EA of residence (as a proxy for individual-level household income) and census tractlevel percentages of black residents, recent immigrants (within the last 5 years), and residents living in the same house over the past 5 years (as a measure of residential stability). Individual-level covariates consisted of baseline age, gender, and measures of baseline patterns of healthcare (continuous variables for number of physician visits and number of admissions to hospitals between 1 January 1992 and 31 December 1993).

\section{Statistical analyses}

Drawing on summary data from Statistics Canada, we first examined the characteristics of public housing residents (aggregated geographically based on public housing postal codes into a single population) by quartile of median household income of the corresponding census tracts. This enabled assessment of the level of quasirandom assignment to projects across census tracts.

The main analyses were based on Cox proportional hazards models, with adjustment of SEs for spatial clustering at the EA level. Individuals with records of the primary diagnosis of interest prior to the start date of follow-up, 1 January 1994, were excluded from the corresponding analyses. Hazard ratios (HRs) for the associations between the quartile of neighbourhood (census tract level) SEP and risk of a diagnosis of each of the four health outcomes and risk of mortality from any cause were estimated. For each outcome, the quartile corresponding to the lowest level of neighbourhood SEP served as the reference group.

Survival time was defined as the time from 1 January 1994 to the date of the primary outcome of interest or the end date of follow-up (31 December 2006), whichever occurred first. For morbidity outcomes, individuals who died prior to the study end date or who remained free of the primary event over the period of follow-up were treated as censored observations. In addition, 
Table 1 Characteristics of residents of public housing postal codes by quartile of 1996 census tract (CT)-level median household income

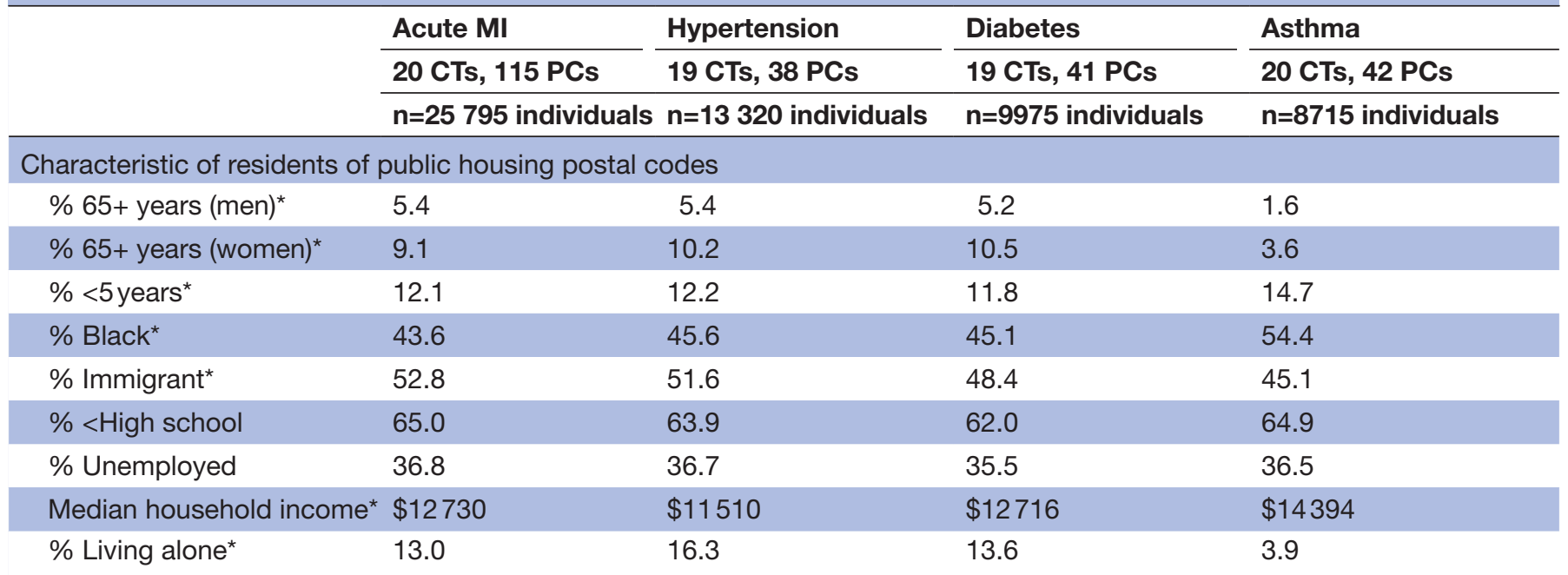

Cutpoints for the quartiles of 1996 CT-level median household income were as follows: \$27000; \$32600; $\$ 39000$.

${ }^{\star} \mathrm{P}<0.01$ for comparison of pooled characteristics of residents of public housing postal codes in Q1-Q3 versus Q4. $\mathrm{Ml}$, myocardial infarction; PCs, postal codes.

individuals who moved into a different postal code after baseline without the primary event by the time of the known change in postal code were treated as censored observations.

All statistical tests were two sided at a significance level of 0.05. All models were estimated using SAS V.9.4.

Datasets were linked using unique, encoded identifiers and analysed at the Institute for Clinical Evaluative Sciences in Toronto, Canada.

\section{RESULTS}

\section{Characteristics of study sample}

Table 1 shows the demographic, socioeconomic, as well as other characteristics of residents of postal codes occupied by public housing projects, by quartile of census tract-level median household income (Q1: $<\$ 27000$, Q2: \$27 000-\$32600, Q3: \$32600-\$39000, Q4: >\$39000). The full cohort was comprised of 29474 individuals residing in 236 postal codes located within 78 unique census tracts. Characteristics were relatively comparable across the three lowest quartiles (Q1-Q3) of median household income, compatible with quasirandom assignment to neighbourhoods. Meanwhile, these characteristics in Q1-Q3 were less similar to those in the highest quartile (Q4), with several characteristics differing among residents of public housing postal codes in Q1-Q3 versus Q4 at the 0.01 significance level (table 1 ). For example, in $\mathrm{Q} 4$, the census tract-level percentage of men aged $65+$ years was $1.6 \%$, compared to $5.2 \%-5.4 \%$ in the other quartiles. The percentage living alone was lower in Q4 (3.9\%) than in the other quartiles $(13.0 \%-16.3 \%)$. Median household income was higher for postal codes in Q4 (\$14394) than in the other quartiles (\$11510-\$12730). In part, these differences could have stemmed from the presence of selection into these neighbourhoods closer to study baseline-for example, if families requested to reside in these neighbourhoods because they viewed them as more desirable.

\section{Associations between neighbourhood SEP and health outcomes}

Table 2 displays the adjusted relative hazards of each separate health outcome (acute MI, diabetes, hypertension, asthma and all-cause mortality) by quartile of census tractlevel median household income based on Cox proportional hazards models. Sample sizes for specific outcomes ranged from 24019 to 28858 adults for acute MI, hypertension, diabetes and all-cause mortality, and 66627 individuals for asthma (aged $4+$ years). The total number of events ranged from 464 incident cases of acute MI to 4625 incident cases of hypertension. Being a resident of a public housing project in the second highest neighbourhood median household income quartile (ie, Q3) was associated with lower relative hazards of acute MI $(\mathrm{HR}=0.79,95 \% \mathrm{CI}$ 0.57 to $1.09 ; \mathrm{P}=0.15)$, death ( $\mathrm{HR}=0.87,95 \%$ CI $0.75-1.02$; $\mathrm{P}=0.08)$ and asthma (HR=0.82, 95\% CI 0.69 to $0.97 ; \mathrm{P}=0.02)$ and with higher relative hazards of hypertension $(\mathrm{HR}=1.09$, 95\% CI 0.99 to 1.19 ; $\mathrm{P}=0.07$ ), compared to living in the lowest neighbourhood median household income quartile, Q1 (table 2). Living in the second lowest neighbourhood median household income quartile (ie, Q2) was also linked inversely and positively to the relative hazards of asthma and hypertension, respectively, compatible with monotonic relationships across the $\mathrm{Q} 1$ through $\mathrm{Q} 3$ quartiles. However, for acute MI and all-cause mortality, living in the Q2 quartile exhibited positive associations (statistically significant for mortality, $\mathrm{HR}=1.17,95 \%$ CI 1.01 to $1.34, \mathrm{P}=0.03$ ), unlike the corresponding associations for living in the Q3 quartile. Meanwhile, the associations corresponding to living in a public housing project in the highest versus lowest quartile of median household income (Q4 vs Q1) were neither 
Table 2 Relative hazards of health outcomes by quartile of 1996 census-tract level median household income based on Cox proportional hazards models

\begin{tabular}{|c|c|c|c|c|c|}
\hline & Acute MI & Hypertension & Diabetes & Asthma & $\begin{array}{l}\text { All-cause } \\
\text { mortality }\end{array}$ \\
\hline & $\mathrm{n}=28858$ & $\mathrm{n}=\mathbf{2 4 0 1 9}$ & $\mathrm{n}=26664$ & $\mathrm{n}=66627$ & $\mathrm{n}=28858$ \\
\hline & 464 cases & 4625 cases & 2436 cases & 2451 cases & 2131 deaths \\
\hline \multicolumn{6}{|c|}{ Neighbourhood level SEP } \\
\hline Q1 (lowest) & 1 (ref) & 1 (ref) & 1 (ref) & 1 (ref) & 1 (ref) \\
\hline Q4 (highest) & $1.05(0.70-1.57)$ & $1.12(0.94-1.32)$ & $1.01(0.84-1.21)$ & $1.03(0.86-1.24)$ & $0.96(0.72-1.27)$ \\
\hline P for trend (Q1-Q4) & 0.49 & 0.12 & 0.95 & 0.55 & 0.26 \\
\hline$P$ for trend (Q1-Q3) & 0.41 & $0.05 \dagger$ & 0.68 & $0.07 \dagger$ & 0.44 \\
\hline
\end{tabular}

All models are adjusted for individual age, gender, baseline number of physician visits and number of admissions to hospitals, EA-level mean household income, CT-level percentages of each of black residents, recent immigrants (within the last 5 years) and residents living in the same house over the previous 5 years and forward sortation area. SEs are adjusted for within-EA clustering. 95\% confidence intervals are shown in parentheses.

*Statistically significant at 0.05 level.

†Statistically significant at 0.10 level.

$\mathrm{CT}$, census tract; EA, enumeration area; MI, myocardial infarction; SEP, socioeconomic position.

consistent in direction nor statistically significant (table 2). There was no significant linear trend across all four quartiles for any of the models. However, across the Q1 through Q3 quartiles, a significant trend was observed at the $10 \%$ level for both hypertension and asthma (table 2).

Table 3 shows similar corresponding adjusted relative hazards of each health outcome by quartile of census-tract level SEP using the combined indicator based on Cox proportional hazards models. Being a resident of a public housing project in the second highest neighbourhood SEP quartile (ie, Q3) was associated with lower relative hazards of acute MI (HR=0.76, 95\% CI 0.54 to $1.07 ; \mathrm{P}=0.11$ ), death (HR=0.86, 95\% CI 0.73 to $1.01 ; \mathrm{P}=0.06)$ and asthma $(\mathrm{HR}=0.80,95 \% \mathrm{CI} 0.67$ to $0.96 ; \mathrm{P}=0.02)$ and with higher relative hazards of hypertension ( $\mathrm{HR}=1.08,95 \%$ CI 0.98 to 1.19; $\mathrm{P}=0.11$ ), compared to living in the lowest neighbourhood SEP quartile, Q1 (table 3). Like the results for neighbourhood median household income, the $\mathrm{Q} 2$ relations for acute MI and mortality were elevated above 1 in contrast to the corresponding Q3 associations. Associations for living in a public housing project in the highest versus lowest quartile of neighbourhood SEP (Q4 vs Q1) were neither consistent nor statistically significant (table 3 ). None of the models indicated a significant linear trend across all four

Table 3 Relative hazards of health outcomes by quartile of 1996 census-tract level SEP (combined indicator) based on Cox proportional hazards models

\begin{tabular}{|c|c|c|c|c|c|}
\hline & Acute MI & Hypertension & Diabetes & Asthma & $\begin{array}{l}\text { All-cause } \\
\text { mortality }\end{array}$ \\
\hline & $\mathrm{n}=28858$ & $n=24019$ & $\mathrm{n}=26664$ & $n=66627$ & $\mathrm{n}=28858$ \\
\hline & 464 cases & 4625 cases & 2436 cases & 2451 cases & 2131 deaths \\
\hline \multicolumn{6}{|c|}{ Neighbourhood level SEP } \\
\hline Q1 (lowest) & 1 (ref) & 1 (ref) & 1 (ref) & 1 (ref) & 1 (ref) \\
\hline Q2 & $1.09(0.83-1.43)$ & $1.05(0.97-1.13)$ & $0.98(0.86-1.12)$ & $0.92(0.79-1.06)$ & $1.12(0.97-1.30)$ \\
\hline Q3 & $0.76(0.54-1.07)$ & $1.08(0.98-1.19)$ & $0.97(0.84-1.12)$ & $0.80(0.67-0.96)^{\star}$ & $0.86(0.73-1.01) \dagger$ \\
\hline Q4 (highest) & $0.99(0.66-1.50)$ & $1.11(0.93-1.31)$ & $0.99(0.82-1.19)$ & $1.01(0.84-1.22)$ & $0.93(0.70-1.25)$ \\
\hline$P$ for trend (Q1-Q4) & 0.37 & 0.15 & 0.82 & 0.44 & 0.19 \\
\hline$P$ for trend (Q1-Q3) & 0.27 & $0.09 \dagger$ & 0.55 & $0.04^{*}$ & 0.30 \\
\hline
\end{tabular}

All models are adjusted for individual age, gender, baseline number of physician visits and number of admissions to hospitals, EA-level mean household income, CT-level percentages of each of black residents, recent immigrants (within the last 5 years) and residents living in the same house over the previous 5 years and forward sortation area. SEs are adjusted for within-EA clustering. 95\% confidence intervals are shown in parentheses.

*Statistically significant at 0.05 level.

†Statistically significant at 0.10 level.

EA, enumeration area; MI, myocardial infarction; SEP, socioeconomic position. 
quartiles. By contrast, across the Q1 through Q3 quartiles, significant trends were observed at the $10 \%$ and $5 \%$ levels for hypertension and asthma, respectively (table 3 ).

In sensitivity analyses, similar results were obtained using the $\%$ below LICO predictor variable (with the highest quartile below LICO as the reference group; data not shown).

In all analyses, being older and being male were associated with higher risks of each health outcome. A higher mean household income (at the EA level) was inversely albeit non-significantly associated with the relative hazards of each of the health outcomes. The absence of a significant association may be attributed to the relatively narrow range of household income within this low-income population.

\section{DISCUSSION}

\section{Principal findings}

In this large, quasiexperimental study, we found new evidence on the estimated impacts of neighbourhood SEP on major chronic diseases and conditions among public housing residents in a large metropolitan area. Based on a 13-year follow-up period, we determined that living in a public housing project in the second highest neighbourhood socioeconomic quartile (Q3) was associated with lower relative hazards of acute MI and significantly associated with lower relative hazards of incident asthma and death from any cause compared to living in the lowest quartile (Q1). Meanwhile, no consistent inverse associations were observed in the highest versus lowest neighbourhood SEP quartile for these outcomes or for the chronic disease outcomes of hypertension and diabetes.

\section{Study strengths and limitations}

Our study had several major strengths, including its reliance on comprehensive administrative databases that allowed for all eligible adult residents of public housing in Toronto to be included, thereby strengthening the generalisability of our findings to that population; its use of longitudinal data, which enabled temporality to support causal inference; its focus on a study population with a relatively narrow range of individual SEP, hence reducing residual confounding by SEP; its employment of health outcomes based on provincial health administrative and vital statistics databases to enhance validity; and its implementation of a novel quasiexperimental design to help minimise endogeneity bias. We further examined the robustness of our findings using alternative SEP measures. Finally, our analyses focused on major chronic diseases and conditions that encompassed a wider set of health outcomes than has been previously investigated in a single study of neighbourhood effects.

Several limitations of our study should also be noted. First, while the quasiexperimental design should have substantially reduced endogeneity bias due to confounding, we cannot entirely rule out the presence of such endogeneity. For example, while we controlled for baseline patterns of healthcare, to the extent that individual/household income (for which EA-level income was an imperfect proxy) and comorbid conditions and their treatments could influence staying versus moving out of the neighbourhood and the outcomes, residual confounding is still possible. While we could not fully eliminate confounding in the lower quartiles (Q1-Q3) of neighbourhood SES, the baseline comparability among their public housing residents suggests that any confounding bias is likely to be smaller in magnitude than in the absence of assignment to public housing projects based on factors other than personal choice. Physical and structural features of neighbourhoods, such as the built environment and air quality, were not specifically controlled for in the models and could further contribute to residual confounding and/or be potential mediators including for the observed asthma associations. Second, moves out of the baseline neighbourhood SEP quartile (with approximately $10 \%-15 \%$ of residents moving to a different quartile over the 13-year follow-up period) as well as differential attrition due to deaths prior to baseline could yield different observed associations than in the absence of such moves/attrition, thereby contributing to selection bias. Such selection could explain the baseline differences in characteristics between the highest quartile $(\mathrm{Q} 4)$ versus lower quartiles (Q1-Q3) of neighbourhood SEP. It could also partly account for the observed null associations for the highest (vs lowest) quartile for all outcomes as well as the non-monotonic associations across Q1 through Q3 seen for acute MI and all-cause mortality. Third, although two OHIP physician service claims were used as the basis for outpatient diagnoses, misclassification of such outcomes is still possible. Finally, while we strengthened the internal validity of our findings through exploiting random variation in neighbourhood SEP of residence, this was done at the expense of external validity. Our findings may not necessarily generalise beyond those living in public housing in urban areas, particularly across much wider ranges of neighbourhood and individual SEP.

\section{Study findings in relation to other studies}

In a 2012 meta-analysis of 18 studies on area-level SEP and all-cause mortality, a significantly lower mortality was found among persons living in areas of higher area-level SEP (relative risk $=0.93,95 \%$ CI 0.91 to 0.96 ). These findings are marginally weaker than what we observed for those living in the second highest (vs lowest) quartile of neighbourhood SEP. Empirical findings for the other health outcomes that we examined have been more mixed. Neighbourhood socioeconomic disadvantage (based on low family incomes, high levels of poverty, public assistance, unemployment, female-headed families, never-married adults and few owner-occupied homes) was not associated with hypertension prevalence $(\mathrm{OR}=1.0,95 \%$ CI 0.1 to 1.2$)$. By contrast, neighbourhood affluence (based on concentrations of people with high education and in professional/managerial occupations) significantly predicted a lower prevalence of hypertension $(\mathrm{OR}=0.7,95 \% \mathrm{CI} 0.6$ to 0.9$) .{ }^{21}$ The few studies on neighbourhood SEP and diabetes/insulin resistance have reported significant inverse associations (eg, Ludwig et $a l^{17}: 21.6 \%$ significantly lower prevalence of high glycated haemoglobin levels among women receiving low-poverty 
housing vouchers vs women in the control group; Rachele et $a l^{22}$ : OR of self-reported diabetes among those in the most vs least disadvantaged neighbourhoods $=1.81,95 \%$ CI 1.15 to 2.83 ) to null associations. ${ }^{23}{ }^{24}$ For asthma, some studies have paradoxically found lower risks among children living in the lowest (vs highest) quartile of neighbourhood SEP ${ }^{25}$ while other studies in adults have found no association. ${ }^{26}{ }^{27}$ Likewise, while we did not examine cholesterol as an outcome, another study observed that in African-American men, lower SEP was associated with higher serum cholesterol in the least socioeconomically disadvantaged neighbourhoods, and with lower serum cholesterol in the most disadvantaged neighbourhoods. ${ }^{28}$ Finally, prospective studies of neighbourhood SEP and incident coronary heart disease and coronary calcification (a marker for underlying coronary heart disease) have generally determined significant positive associations with moderate to strong relative risks above $1.5 .^{3}{ }^{1929-32}$

Our null findings for associations among individuals living in public housing in the highest versus lowest quartile of neighbourhood SEP are compatible with previous evidence showing that low-income individuals living in low-income neighbourhoods experience lower mortality rates than low-income individuals in high-income neighbourhoods. ${ }^{33-37}$ Our findings of a positive relation between higher neighbourhood SEP and hypertension are also compatible with past evidence of associations between neighbourhood SEP and worse CHD risk factor profiles for low-income individuals in high-income neighbourhoods, although the specific association observed in a prior study was with cholesterol rather than hypertension. ${ }^{28}$ Such findings may possibly reflect the adverse health effects of status incongruity, whereby feelings of relative deprivation and social exclusion for low-income individuals living in high-income neighbourhoods may generate psychosocial stress. ${ }^{37}$ Furthermore, as supported by a US survey of public housing residents, given a wider set of incomes within these neighbourhoods, such low-income individuals may find fewer opportunities to form close-knit networks with other low-income individuals that can serve to provide beneficial social support and other resources to buffer against the adverse effects of social inequalities. ${ }^{37-39}$ Finally, it is possible that with higher quality healthcare services including primary healthcare being available in higher SEP neighbourhoods, such neighbourhoods could be linked to a higher probability of being medically diagnosed with hypertension.

\section{Meaning of the study: implications}

Our study advances knowledge on the impacts of neighbourhood socioeconomic environments on all-cause mortality and several major chronic diseases and conditions. Through implementing a novel quasiexperimental design, our study overcomes major limitations of many observational studies to date, in particular endogeneity bias. We found evidence to support protective influences of neighbourhood SEP on incident asthma. Should these associations be truly causal, our findings suggest that raising the SEP of the neighbourhoods where low-income individuals reside (such as through neighbourhood revitalisation programmes) or relocating low-income residents to neighbourhoods with higher SEP (such as through housing choice voucher programmes) may serve as means to alleviate the burden of selected chronic conditions such as asthma. At the same time, our evidence points to possible limits to the health benefits of raising the neighbourhood SEP of low-income residents above a certain threshold due to status incongruity.

\section{Unanswered questions and future research}

Unpacking the behavioural, psychosocial and biological mechanisms for the observed protective association for asthma would advance our understanding of these apparent effects and provide further support for the presence of causal associations. Likewise, future epidemiological studies that implement quasiexperimental study designs to exploit the random variation in neighbourhood of residence would help to confirm our findings. Finally, the observed null associations for public housing residents in the highest (vs lowest) quartile of neighbourhood SEP warrant further inquiry. Exploring their underlying mechanisms including the potentially contrasting benefits of neighbourhood affluence and harms of relative deprivation could aid in identifying optimal points of intervention.

In summary, this quasiexperimental study provides new evidence compatible with protective influences of higher neighbourhood SEP on health, particularly for the risk of the chronic condition of asthma. Programmes to improve neighbourhood SEP or relocate low-income residents to higher SEP neighbourhoods in urban areas may serve as possible upstream levers to improve population health and bridge socioeconomic disparities in health.

\section{Author affiliations}

${ }^{1}$ Department of Health Sciences, Northeastern University, Boston, Massachusetts, USA

${ }^{2}$ Department of Social and Behavioral Sciences, EHESP School of Public Health, Sorbonne Paris Cité, Paris Descartes University, Paris, France

${ }^{3}$ Institute for Clinical Evaluative Sciences, Toronto, Ontario, Canada

${ }^{4}$ Centre for Urban Health Solutions, Li Ka Shing Knowledge Institute, St. Michael's Hospital, Toronto, Ontario, Canada

${ }^{5}$ Family and Community Medicine, St. Michael's Hospital and University of Toronto, Toronto, Ontario, Canada

${ }^{6}$ Institute of Health Policy, Management and Evaluation, Faculty of Medicine, University of Toronto, Toronto, Ontario, Canada

${ }^{7}$ BMZ Analytics, Toronto, Ontario, Canada

${ }^{8}$ Department of Social and Behavioral Sciences, Harvard T.H. Chan School of Public Health, Boston, Massachusetts, USA

${ }^{9}$ Department of Economics, University of Toronto, Toronto, Ontario, Canada

Contributors DK conceived and designed the study. DK, BZ and PO gathered data, and $R G$ facilitated access to data. DK and BZ performed data analyses, and DK supervised the analyses by BZ. DK drafted the manuscript. DK, RG, IK and P0 revised the manuscript for important intellectual content. DK is guarantor.

Funding This study was funded by a grant to DK through the Robert Wood Johnson Foundation. DK is supported by grant R01 HL138247 through the National Heart, Lung, and Blood Institute at the U.S. National Institutes of Health. RG is supported as a Clinician Scientist in the Department of Family and Community Medicine at St. Michael's Hospital and the University of Toronto. This study was also supported by the Institute for Clinical Evaluative Sciences (ICES), which is funded by an annual grant from the Ontario Ministry of Health and Long-Term Care (MOHLTC). 
Disclaimer The opinions, results and conclusions reported in this paper are those of the authors and are independent from all funding sources. No endorsement by the National Institutes of Health, Robert Wood Johnson Foundation, ICES, or the Ontario MOHLTC is intended or should be inferred.

Competing interests None declared.

Patient consent Not required.

Ethics approval Research Ethics Board at Sunnybrook Health Sciences Centre.

Provenance and peer review Not commissioned; externally peer reviewed.

Data sharing statement Results from sensitivity analyses that use the percentage below LICO as the predictor variable are available from the authors upon request.

Open Access This is an Open Access article distributed in accordance with the Creative Commons Attribution Non Commercial (CC BY-NC 4.0) license, which permits others to distribute, remix, adapt, build upon this work non-commercially, and license their derivative works on different terms, provided the original work is properly cited and the use is non-commercial. See: http://creativecommons.org/ licenses/by-nc/4.0/

(C) Article author(s) (or their employer(s) unless otherwise stated in the text of the article) 2018. All rights reserved. No commercial use is permitted unless otherwise expressly granted.

\section{REFERENCES}

1. Riva M, Gauvin L, Barnett TA. Toward the next generation of research into small area effects on health: a synthesis of multilevel investigations published since July 1998. J Epidemiol Community Health 2007;61:853-61.

2. Macintyre S, Ellaway A. Neighborhoods and health: an overview. In: Kawachi I, Berkman LF, eds. Neighborhoods and health. New York: Oxford University Press, 2003:20-44.

3. Kim D, Diez Roux AV, Kiefe Cl, et al. Do neighborhood socioeconomic deprivation and low social cohesion predict coronary calcification?: the CARDIA study. Am J Epidemiol 2010;172:288-98.

4. Kubzansky LD, Subramanian SV, Kawachi I, et al. Neighborhood contextual influences on depressive symptoms in the elderly. $\mathrm{Am} J$ Epidemiol 2005;162:253-60.

5. Smith GD, Hart C, Watt G, et al. Individual social class, area-based deprivation, cardiovascular disease risk factors, and mortality: the Renfrew and Paisley Study. J Epidemiol Community Health 1998;52:399-405.

6. Waitzman NJ, Smith KR. Phantom of the area: poverty-area residence and mortality in the United States. Am J Public Health 1998;88:973-6.

7. Kawachi I, Berkman LF. Introduction. In: Kawachi I, Berkman LF, eds. Neighborhoods and Health. New York: Oxford University Press, 2003:1-19.

8. Cubbin C, Hadden WC, Winkleby MA. Neighborhood context and cardiovascular disease risk factors: the contribution of material deprivation. Ethn Dis 2001;11:687-700.

9. Kim D. Blues from the neighborhood? Neighborhood characteristics and depression. Epidemiol Rev 2008;30:101-17.

10. Chaix $B$, Ducimetière $P$, Lang $T$, et al. Residential environment and blood pressure in the PRIME Study: is the association mediated by body mass index and waist circumference? J Hypertens 2008;26:1078-84.

11. Krieger N. Why epidemiologists cannot afford to ignore poverty. Epidemiology 2007;18:658-63.

12. Diez Roux AV, Borrell LN, Haan M, et al. Neighbourhood environments and mortality in an elderly cohort: results from the cardiovascular health study. J Epidemiol Community Health 2004;58:917-23.

13. Oakes JM, Kaufman JS, eds. Methods in social epidemiology. San Francisco, CA: Jossey-Bass, 2006.

14. Rosenbaum J, DeLuca S, Miller S. The long-term effects of residential mobility on AFDC receipt: studying the Gautreaux Program with administrative data: Mimeo, Northwestern University, 1999.

15. Popkin SJ, Rosenbaum JE, Meaden PM. Labor market experiences of low-income black women in middle-class suburbs: evidence from a survey of gautreaux program participants. J Policy Anal Manag 1993;12:556-73.
16. Kling JR, Liebman JB, Katz LF. Experimental analysis of neighborhood effects. Econometrica 2007;75:83-119.

17. Ludwig J, Sanbonmatsu L, Gennetian L, et al. Neighborhoods, obesity, and diabetes--a randomized social experiment. $N$ Engl $J$ Med 2011;365:1509-19.

18. Oreopoulos P. The long-run consequences of living in a poor neighborhood. Q J Econ 2003;118:1533-75.

19. Diez Roux AV, Merkin SS, Arnett D, et al. Neighborhood of residence and incidence of coronary heart disease. N Engl J Med 2001;345:99-106.

20. Hux J, Tang M. Patterns of prevalence of incidence of diabetes. Diabetes in Ontario. An ICES Practice Atlas, Module 1, 2002. http:// www.ices.on.ca

21. Morenoff JD, House JS, Hansen BB, et al. Understanding social disparities in hypertension prevalence, awareness, treatment, and control: the role of neighborhood context. Soc Sci Med 2007;65:1853-66.

22. Rachele JN, Giles-Corti B, Turrell G. Neighbourhood disadvantage and self-reported type 2 diabetes, heart disease and comorbidity: a cross-sectional multilevel study. Ann Epidemiol 2016;26:146-50.

23. Diez Roux AV, Jacobs DR, Kiefe Cl. Neighborhood characteristics and components of the insulin resistance syndrome in young adults: the coronary artery risk development in young adults (CARDIA) study. Diabetes Care 2002;25:1976-82.

24. Auchincloss AH, Diez Roux AV, Brown DG, et al. Association of insulin resistance with distance to wealthy areas: the multi-ethnic study of atherosclerosis. Am J Epidemiol 2007;165:389-97.

25. Juhn YJ, Sauver JS, Katusic S, et al. The influence of neighborhood environment on the incidence of childhood asthma: a multilevel approach. Soc Sci Med 2005;60:2453-64.

26. Cagney KA, Browning CR. Exploring neighborhood-level variation in asthma and other respiratory diseases. J Gen Intern Med 2004;19:229-36.

27. Coogan PF, Castro-Webb N, Yu J, et al. Neighborhood and individual socioeconomic status and asthma incidence in African American women. Ethn Dis 2016;26:113.

28. Diez-Roux AV, Nieto FJ, Muntaner C, et al. Neighborhood environments and coronary heart disease: a multilevel analysis. Am J Epidemiol 1997:146:48-63.

29. Sundquist K, Winkleby M, Ahlén $\mathrm{H}$, et al. Neighborhood socioeconomic environment and incidence of coronary heart disease: a follow-up study of 25,319 women and men in Sweden. Am J Epidemiol 2004;159:655-62.

30. Stjärne MK, Fritzell J, De Leon AP, et al. Neighborhood socioeconomic context, individual income and myocardial infarction. Epidemiology 2006;17:14-23.

31. Chaix B, Rosvall M, Merlo J. Neighborhood socioeconomic deprivation and residential instability: effects on incidence of ischemic heart disease and survival after myocardial infarction. Epidemiology 2007;18:104-11.

32. Merlo J, Ohlsson H, Chaix B, et al. Revisiting causal neighborhood effects on individual ischemic heart disease risk: a quasiexperimental multilevel analysis among Swedish siblings. Soc Sci Med 2013;76:39-46.

33. Roos NP, Mustard CA. Variation in health and health care use by socioeconomic status in Winnipeg, Canada: does the system work well? Yes and no. Milbank Q 1997;75:89-111.

34. Veugelers PJ, Yip AM, Kephart G. Proximate and contextual socioeconomic determinants of mortality: multilevel approaches in a setting with universal health care coverage. Am J Epidemiol 2001;154:725-32.

35. Wen M, Christakis NA. Neighborhood effects on posthospitalization mortality: a population-based cohort study of the elderly in Chicago. Health Serv Res 2005;40:1108-27.

36. Yen IH, Kaplan GA. Neighborhood social environment and risk of death: multilevel evidence from the Alameda County Study. Am J Epidemiol 1999;149:898-907.

37. Albor C, Uphoff EP, Stafford M, et al. The effects of socioeconomic incongruity in the neighbourhood on social support, self-esteem and mental health in England. Soc Sci Med 2014;111:1-9.

38. Schwartz H, Burkhauser S, Griffin BA, et al. Do the Joneses help you keep up? A natural experiment in exposure to nonpoor neighbors. Hous Policy Debate 2015;25:320-52.

39. Uphoff EP, Pickett KE, Cabieses B, et al. A systematic review of the relationships between social capital and socioeconomic inequalities in health: a contribution to understanding the psychosocial pathway of health inequalities. Int J Equity Health 2013;12:54. 
Correction: Neighbourhood socioeconomic position and risks of major chronic diseases and all-cause mortality: a quasiexperimental study

Kim D, Glazier RH, Zagorski B, et al. Neighbourhood socioeconomic position and risks of major chronic diseases and all-cause mortality: a quasi-experimental study. BMJ Open 2018;8:e18793. doi: 10.1136/bmjopen-2017-018793.

The previous version of this manuscript contains an error in table 1 column headings.

The column headings should read respectively as:

Q1 (lowest income CTs)

Q2

Q3

Q4 (highest income CTs)

Instead of:

Acute MI

Hypertension

Diabetes

Asthma

Open access This is an open access article distributed in accordance with the Creative Commons Attribution Non Commercial (CC BY-NC 4.0) license, which permits others to distribute, remix, adapt, build upon this work non-commercially, and license their derivative works on different terms, provided the original work is properly cited, appropriate credit is given, any changes made indicated, and the use is non-commercial. See: http://creativecommons.org/licenses/by-nc/4.0/.

(C) Author(s) (or their employer(s)) 2019. Re-use permitted under CC BY-NC. No commercial re-use. See rights and permissions. Published by BMJ.

BMJ Open 2019;9:e018793corr1. doi:10.1136/bmjopen-2017-018793corr1

A) Check for updates 